\title{
Performance Analysis of Wireless Mesh Backhauling Using Intelligent Reflecting Surfaces
}

\author{
M. Al-Jarrah, Member, IEEE, E. Alsusa, Senior Member, IEEE, A. \\ Al-Dweik, Senior Member, IEEE, and M.-S. Alouini, Fellow, IEEE
}

\begin{abstract}
This paper considers the deployment of intelligent reflecting surfaces (IRSs) technology for wireless multi-hop backhauling of multiple basestations (BSs) connected in a mesh topology. The performance of the proposed architecture is evaluated in terms of outage and symbol error probability in Rician fading channels, where closed-form expressions are derived and demonstrated to be accurate for several cases of interest. The analytical results corroborated by simulation, show that the IRS-mesh backhauling architecture has several desired features that can be exploited to overcome some of the backhauling challenges, particularly the severe attenuation at high frequencies. For example, using IRS with four elements, $N=4$, provides a symbol error rate of about $10^{-5}$ at a signal-to-noise ratio of about $0 \mathrm{~dB}$, even for a large number of hops. Moreover, the obtained analytical results corroborated by Monte Carlo simulation show that the gain obtained by increasing $N$ decreases significantly for $N>5$. For example, increasing $N$ from 1 to 2 provides about 8 dB of gain, while the increase from 3 to 4 provides about $4 \mathrm{~dB}$. Moreover, the degradation caused by the relaying process becomes negligible when the number of IR elements $N=3$.
\end{abstract}

\section{Index Terms}

6G, wireless backhauling, mesh backhauling, intelligent reflecting surfaces (IRSs), symbol error rate, outage probability, Rician channel.

M. A. Al-Jarrah and E. Alsusa are with the School of Electrical and Electronic Engineering, University of Manchester, Manchester M13 9PL, U.K. (e-mail: \{mohammad.al-jarrah,e.alsusa\}@manchester.ac.uk).

A. Al-Dweik is with Center for Cyber Physical Systems (C2PS), Khalifa University, Abu Dhabi, P.O.Box 127788, UAE. $\mathrm{He}$ is also with the Department of Electrical and Computer Engineering, Western University, London, ON, Canada. (e-mail: dweik@fulbrightmail.org).

M.-S. Alouini is with King Abdullah University of Science and Technology (KAUST), Thuwal, Makkah Province, Kingdom of Saudi Arabia, (E-mail: slim.alouini@kaust.edu.sa). 


\section{INTRODUCTION}

Current wireless communication networks such as the fourth and fifth generations, (4G) and (5G) respectively, have to support more than 6 billion users with a considerable proportion running applications that require high data rates [1], [2], that severely strain the available spectrum. Moreover, the demand for data services is continuously increasing as shown by the latest statistics of wireless networks, which revealed that data traffic for mobile users grew $68 \%$ in 2019 , reaching 38 Exabytes (EB) per month as compared to 27 EB per month in 2018 [1], [2]. Ericsson predicts that the mobile data traffic will show an upsurge of $27 \%$ annually till 2025 . Network densification $(\mathrm{NeDe})$ is one of the prominent techniques that can be used to improve the capacity of wireless networks, and it is an integral part of the 5G architecture [3]. The main concept of $\mathrm{NeDe}$ is to deploy a large number of small cells with low power to complement the macro cell functionality in areas with poor signal quality, such as indoor environments and urban areas with tall buildings [4], [5]. The term small-cell covers a variety of cell types such as mini-cells, micro-cells, picocells, femto-cells, and mobile-small cell. While indoor small-cell backhauling is typically based on wired technologies, outdoor small cells backhauling is mostly performed through wireless technologies, which may increase the marco cell backhauling traffic rate to several giga bits per second (Gpbs) [6].

Wireless backhauling is an integral technology of the current $5 \mathrm{G}$, future sixth generation (6G) and $6 \mathrm{G}^{+}$cellular communication networks. The main advantages of wireless backhauling over traditional wired backhauling are the implementation flexibility, cost efficiency, and lowcomplexity maintenance processes. Moreover, integrated access and backhauling is considered as a promising approach to reduce the deployment cost in ultra-dense networks [7]-[9]. Therefore, wireless backhauling has attracted extensive research attention to develop efficient backhauling solutions. One of the prominent efforts is the Telecom Infra Project (TIP), which consists of more than 500 member organizations that devote their efforts to accelerate the development of new infrastructure solutions for future networks [10]-[14]. Wireless backhauling can be realized with several network topologies such as ring, tree and mesh, with the later being the most attractive because it provides backhauling with low-cost, flexible configuration, maintainable, and long distance coverage[15]-[18]. However, wireless backhauling suffers from some limitations 
as compared to optical fiber, particularly the relatively low capacity, disruptive interference, and severe signal attenuation at high frequencies. Therefore, several researchers have proposed transmission schemes and technologies to overcome one or more of the wireless backhauling limitations. Examples for such solutions include millimeter-wave (mmWave) transmission, freespace optical (FSO), interference management protocols [19]-[21] and massive multiple-inputmultiple-output (mMIMO) [22]-[26].

The lack of spectrum supporting wide channel bandwidths has been identified as a potential bottleneck for wireless backhaul. This has given spectrum administrators the opportunity to introduce wider channels in currently used frequencies. An additional possibility is to open new frequency bands such as the $90 \mathrm{GHz}$ band [6]. The European Telecommunications Standards Institute (ETSI) and TIP recommend mmWave communications as a core technology [10]-[12]. Realistic experiments have been conducted for one giga bits per second (Gbps) average peak user throughput for a maximum range of $250 \mathrm{~m}$ [11]. In addition, the TIP backhaul team has proposed different methods for assessing the performance of mmWave, and provided a guidance for the installation process [13], [14].

Intelligent reflecting surfaces (IRSs), also called metasurfaces, have been introduced recently with the aim of controlling the propagation medium to enhance the quality of service (QoS) by boosting the energy and spectral efficiencies of wireless networks. The IRS technology is expected to play a significant role in the future, where smartness, energy efficiency and spectral efficiency are the main requirements for the forthcoming wireless networks. IRS applies a large number of passive antenna elements which introduce a phase-shift to the received signals, and reflect them back to the destination. For efficient transmission, multiple reflectors are used for a certain destination, and the introduced phase shifts are selected to ensure that the reflected signals add coherently in the channel. As a result, the signal-to-noise ratio (SNR) increases considerably, and consequently, the spectral efficiency is boosted [27]-[46].

\section{A. Related Work}

IRS has recently attracted extensive research attention. For example, the authors of [27], [28] presented detailed IRS technology overview, and discussed state-of-art solutions and theoreti- 
cal performance limits. Energy-efficient approaches for the transmit power allocation and the phase shifts of the IRS elements is introduced in [29], where an accurate model for IRS power consumption is presented. A realistic implementation in outdoor environments has shown that the methods proposed in [29] for IRS power allocation may provide up to 300\% higher energy efficiency when compared to multi-antenna and amplify-and-forward systems. Joint active and passive beamforming is considered in [30], where some recommendations are provided for optimal deployment. Other research efforts are dedicated to study the performance of IRS with other existing signalling and communication technologies such as index modulation (IM) [31], spaceshift-keying (SSK) [32], and non-orthogonal multiple access (NOMA) [46]. In [33], IRS assisted MIMO is investigated, where efficient algorithms for the phase shifts at the IRS and precoding at the transmitter are proposed to minimize the symbol error rate (SER). Since the optimum values for the phase shifts depend on the instantaneous channel side information (CSI), channel modeling and estimation are considered in [34]-[36]. In [47], the far-field pathloss model is derived for IRS based links using optical physics techniques, and it is shown that each reflecting element acts as a diffuse scatterer. A practical IRS implementation is introduced in [48], where a high-gain and low-cost IRS with 256 reflecting elements is designed, in which positive intrinsic negative (PIN) diodes are used to design 2-bit phase shifters, and it was shown that a gain of $19.1 \mathrm{dBi}$ can be achieved using mmWave.

\section{B. Motivation and Contribution}

Future mobile networks are expected to confine more of the radio communication needs of current users, and support many new users and industries, with applications that require ultra high data rates. Examples for such applications include holographic communication, online gaming, and streaming of super high definition immersive 3D videos, etc. To be able to support such applications for a large number of users, the network should be able to handle traffic volumes in the range of Tbytes/s per square, coming from a massive simultaneous connections [6]. Consequently, the backhaul traffic between the base stations (BSs) is expected to experience enormous data rate and traffic volume increase. Therefore, the design of a backhaul that satisfies such scenarios is indispensable. Although IRSs have been considered widely in the recent literature [27], [28], to 


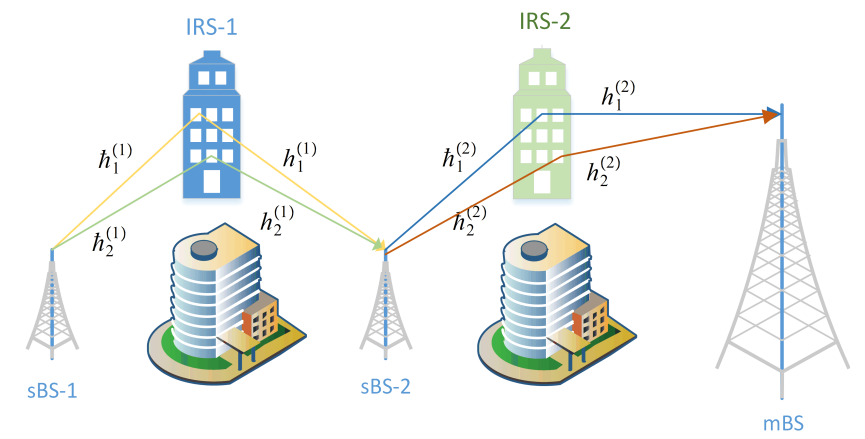

Fig. 1. Example for a two-hop backhauling using IRS.

the best of the authors' knowledge, there is no work reported in the literature that considers the application of IRS to wireless mesh backhauling with multiple hops, where BSs relay the traffic until it arrives to the core network. Because wireless backhauling typically requires lineof-sight (LoS) connectivity to avoid severe signal fading, the channel gain is assumed to follow the Rician fading model, where an accurate approximation for the probability density function (PDF) of the received SNR is derived. Moreover, analytical expressions are derived for the SER and outage probability (OP). The obtained analytical results corroborated by Monte Carlo simulation demonstrate that the gain achieved by increasing the number of IRS elements is inversely proportional to the number of IRS elements $N$, particularly for $N>5$. Moreover, the degradation caused by the relaying process becomes negligible when the number of IRS elements is more than 3 .

\section{Paper organization}

The rest of the paper is organized as follows. Section II presents the system model with IRS based wireless backhauling. A derivation for an accurate approximation for the PDF of the received SNR per hop is introduced in Section III. The derivations for the SER and OP for each hop are provided in Sections IV and V, respectively. The derivations of the SER and OP are extended to multiple hop mesh backhauling in Section VI. Section VII shows the numerical results for the proposed system whereas the conclusions and future work are provided in Section VIII. 


\section{SYSTEM MODEL}

This work considers a heterogenous wireless network where a macro cell is overlaid with multiple small cells. The macro BS (mBS) and small-cells BS (sBS) are configured in a mesh topology. The sBS cooperate, act as relays, to route the backhauling traffic to the mBS, and then to the core network. Therefore, the traffic of a particular sBs may arrive to the mBS through multiple hops, where the signal in each hop is decoded and forwarded to the next sBS, or to the mBS. To provide LoS links between adjacent sBSs, IRS panels, each of which consists of $N$ reflecting elements, are placed between each two BSs. The backhaul links are realized using a single broadband antenna at each BS. Using the region three-dimensional maps, advanced ray tracing techniques [65] can be used to predict the CSI between adjacent BSs and the IRS, which allow selecting the optimum location for the IRS panels'. The channel fading is considered to be Rician to capture the LoS signal component and other small reflections from the surrounding environment. The LoS is considered only between the BSs and the IRS, while the direct path between BSs is typically blocked by large buildings or other obstacles.

In each hop, a sBS transmits its backhaul traffic to one of the adjacent sBSs, or to the $\mathrm{mBS}$, through an IRS panel, where each reflecting element introduces a phase shift $\varphi_{n}$ and amplitude gain $\beta_{n}$. The process is repeated $L$ times until the data arrive at the corenetwork or mBS. It is worth noting that an IRS panel can be shared by multiple BSs simultaneously, where the available reflecting elements can be assigned to BSs that have LoS link with the IRS panel. However, an IRS element can be assigned only to one BS to avoid interference.

\section{A. Single hop received signal model}

In this work, we initially consider the single hop scenario, and then the model is extended to multiple hops. Consequently, the hop index is dropped unless it is necessary to include it. Given that a particular sBS transmits a data symbol $s$, the signal reflected from the $n$th IRS element can be expressed as

$$
x_{n}=\sqrt{P_{0}} H_{n} \hbar_{n} s, n \in\{1,2, \ldots, N\}
$$


where $H_{n}=\beta_{n} \mathrm{e}^{j \varphi_{n}}, \varphi_{n}$ is the phase shift and $\beta_{n} \leq 1$ is the reflection coefficient of the $n$th IRS element, $P_{0}$ is the sBS transmission power, and the channel coefficient $\hbar_{n} \triangleq\left|\hbar_{n}\right| \mathrm{e}^{j \theta_{n}} \sim$ $\mathcal{C N}\left(m_{n}, 2 \sigma_{n}^{2}\right)$. Due to the LoS signal, the average channel fading $m_{n} \neq 0$, the PDF of the channel envelop $\alpha_{n} \triangleq\left|\hbar_{n}\right|$ can be considered Rician [49],

$$
f\left(\alpha_{n}\right)=\frac{2\left(1+K_{n}\right)}{\Omega_{n}} \alpha_{n} \mathrm{e}^{-K_{n}} \mathrm{e}^{-\frac{\left(1+K_{n}\right)}{\Omega_{n}} \alpha^{2}} I_{0}\left(2 \alpha_{n} \sqrt{\frac{K_{n}\left(1+K_{n}\right)}{\Omega_{n}}}\right)
$$

where $I_{0}(\cdot)$ is the modified Bessel function of the first kind and zero order, $\Omega_{n} \triangleq \mathrm{E}\left[\alpha_{n}^{2}\right]=$ $\mu_{n}^{2}+2 \sigma_{n}^{2}, \mu_{n}=\left|m_{n}\right|$ and $K_{n}=\frac{\mu_{n}^{2}}{2 \sigma_{n}^{2}}$ is the Rician factor that determines the link quality, $K_{n} \in(0$, $\infty)$. For small values of $K_{n}$, the channel fading becomes severe, which indicates that the LoS signal component is weak. Strong links are typically achieved when $K_{n} \gtrsim 9 \mathrm{~dB}$ [56], [57]. The first moment of $\alpha_{n}$ with parameters $K_{n}$ and $\Omega_{n}$ is given by [54]

$$
\mathrm{E}\left[\alpha_{n}\right]=\frac{1}{2} \sqrt{\frac{\pi \Omega_{n}}{1+K_{n}}}{ }_{1} F_{1}\left(-\frac{1}{2}, 1,-K_{n}\right)
$$

where ${ }_{1} F_{1}(\cdot, \cdot, \cdot)$ is the confluent hypergeometric function of the first kind.

In the reflecting phase, the signal will go through a second fading channel $h_{n}$ before arriving to the destination BS. Therefore, the received signal at the destination BS is given by

$$
\begin{aligned}
y & =\sqrt{P_{0}} \sum_{n=1}^{N} \beta_{n} h_{n} \hbar_{n} \mathrm{e}^{j \varphi_{n}} s+w \\
& =\sqrt{P_{0}} \sum_{n=1}^{N} \beta_{n}\left|\hbar_{n}\right|\left|h_{n}\right| \mathrm{e}^{j\left(\theta_{n}+\zeta_{n}+\varphi_{n}\right)} s+w .
\end{aligned}
$$

where the channel coefficient $h_{n} \triangleq\left|h_{n}\right| \mathrm{e}^{j \zeta_{n}} \sim \mathcal{C N}\left(\bar{m}_{n}, 2 \bar{\sigma}_{n}^{2}\right)$ and $w$ is the additive white Gaussian noise $(\mathrm{AWGN}), w \sim \mathcal{C N}\left(0, \sigma_{w}^{2}\right)$. By selecting $\varphi_{n}=-\left(\theta_{n}+\zeta_{n}\right)$, all the reflected signal components are added coherently in the channel, and thus, the received signal BS can be rewritten as

$$
y=\sqrt{P_{0}} \sum_{n=1}^{N} \beta_{n} \varpi_{n} s+w
$$

where $\left|\hbar_{n}\right|\left|h_{n}\right| \triangleq \varpi_{n}$. Therefore, $\varpi_{n}$ is formed by the product of two Rician random variables, 
and hence, its PDF is given by [66, eq. 6.67]

$$
f_{\varpi_{n}}\left(\varpi_{n}\right)=\frac{\mathrm{e}^{-\left(K_{n}+\bar{K}_{n}\right)}}{\sigma_{n}^{2} \bar{\sigma}_{n}^{2}} \varpi_{n} \sum_{i=0}^{\infty} \sum_{l=0}^{\infty} \frac{1}{(i ! l !)^{2}}\left(\frac{K_{n}}{2 \sigma_{n}^{2}}\right)^{i}\left(\frac{\bar{K}_{n}}{2 \bar{\sigma}_{n}^{2}}\right)^{l}\left(\frac{\sigma_{n}}{\bar{\sigma}_{n}}\right)^{i-l} \varpi_{n}^{i+l} \mathcal{K}_{i-l}\left(\frac{\varpi_{n}}{\sigma_{n} \bar{\sigma}_{n}}\right)
$$

where $\bar{K}$ is the Rician factor for channel $h_{n}$ and $\mathcal{K}_{m}(\cdot)$ is the modified Bessel function of the second kind with order $m$.

\section{SNR DISTRIBUTION}

To derive the SER and OP, the SNR distribution should be evaluated. Towards this goal, the following two subsections present the PDF derivation of the instantaneous SNR at the destination BS for a single and multiple reflectors, respectively.

\section{A. Single reflector $(N=1)$}

Based on the received signal in (5), the instantaneous SNR of the received signal at the BS for a signal reflected from the $n$th reflector, can be written as

$$
\gamma_{n}=\mathcal{P} \beta_{n}^{2} \varpi_{n}^{2}
$$

where $\mathcal{P} \triangleq P_{0} / \sigma_{w}^{2}$. Using $f_{\varpi_{n}}\left(\varpi_{n}\right)$ given in (6), the PDF of $\gamma_{n}$ can be obtained by applying random variable transformation. Consequently, $f_{\gamma_{n}}\left(\gamma_{n}\right)$ can be written as

$$
f_{\gamma_{n}}\left(\gamma_{n}\right)=C_{n} \sum_{i=0}^{\infty} \sum_{l=0}^{\infty} D_{i, l}^{n} \gamma_{n}^{\frac{i+l}{2}} \mathcal{K}_{i-l}\left(E_{n} \sqrt{\gamma_{n}}\right)
$$

where

$$
\begin{aligned}
E_{n} & =\frac{2}{\beta_{n}} \sqrt{\frac{\left(1+K_{n}\right)\left(1+\bar{K}_{n}\right)}{\Omega_{n} \bar{\Omega}_{n} \mathcal{P}}} \\
C_{n} & =\frac{E_{n}^{2}}{2} \mathrm{e}^{-\left(K_{n}+\bar{K}_{n}\right)} \\
D_{i, l}^{n} & =\frac{K_{n}^{i} \bar{K}_{n}^{l}}{(i ! l !)^{2}}\left(\frac{E_{n}}{2}\right)^{i+l} .
\end{aligned}
$$




\section{B. Multiple Reflectors $(N>1)$}

Based on the received signal in (5), the instantaneous SNR for $N>1$ can be written as

$$
\gamma=\left(\sqrt{\mathcal{P}} \sum_{n=1}^{N} \beta_{n} \varpi_{n}\right)^{2} .
$$

Unlike the single reflector case, deriving a closed-form expression for the PDF of $\gamma$ is intractable. Consequently, an approximate PDF will be derived to enable analytical performance evaluation. To this end, the cascaded channels for the $n$th reflector can be written as $Z_{n}=$ $\hbar_{n} h_{n} \triangleq Z_{n}^{I}+j Z_{n}^{Q}$, where $Z_{n}^{I}=\hbar_{n}^{I} h_{n}^{I}-\hbar_{n}^{Q} h_{n}^{Q}$ and $Z_{n}^{Q}=\hbar_{n}^{I} h_{n}^{Q}+\hbar_{n}^{Q} h_{n}^{I},\left\{\hbar_{n}^{I}, \hbar_{n}^{Q}, h_{n}^{I}, h_{n}^{Q}\right\} \sim$ $\mathcal{N}\left(\left\{\bar{m}_{n}^{I}, \bar{m}_{n}^{Q}, m_{n}^{I}, m_{n}^{Q}\right\},\left\{\bar{\sigma}_{n}^{2}, \bar{\sigma}_{n}^{2}, \sigma_{n}^{2}, \sigma_{n}^{2}\right\}\right)$. In general, the product of two independent Gaussian random variables $X \sim \mathcal{N}\left(m_{X}, \sigma_{X}^{2}\right)$ and $Y \sim \mathcal{N}\left(m_{Y}, \sigma_{Y}^{2}\right)$ is not Gaussian [50]. However, if $\left\{\frac{\mu_{X}}{\sigma_{X}}, \frac{\mu_{Y}}{\sigma_{Y}}\right\} \gg 1$, then PDF of the product can be approximated by $\mathcal{N}\left(m_{X} m_{Y}, m_{X}^{2} \sigma_{Y}^{2}+m_{Y}^{2} \sigma_{X}^{2}\right)$ [51], [52]. For Rician fading channels, such condition can be satisfied when the Rician factors $\{\bar{K}, K\} \gg 1 \mathrm{~dB}$. Consequently, the PDFs of $Z_{n}^{I}$ and $Z_{n}^{Q}$ can be approximated as $Z_{n}^{I} \sim$ $\mathcal{N}\left(m_{Z_{n}^{I}}, \sigma_{Z_{n}}^{2}\right)$ and $Z_{n}^{Q} \sim \mathcal{N}\left(m_{Z_{n}^{Q}}, \sigma_{Z_{n}}^{2}\right)$. Given that $\sigma_{n}^{I}=\sigma_{n}^{Q}=\sigma_{n}$ and $\bar{\sigma}_{n}^{I}=\bar{\sigma}_{n}^{Q}=\bar{\sigma}_{n}$, the statistics of $Z_{n}^{I}$ and $Z_{n}^{Q}$ are given by

$$
\begin{aligned}
m_{Z_{n}^{I}} & =m_{n}^{I} \bar{m}_{n}^{I}-m_{n}^{Q} \bar{m}_{n}^{Q} \\
\sigma_{Z_{n}^{I}}^{2} & =\mu_{n}^{2} \bar{\sigma}_{n}^{2}+\bar{\mu}_{n}^{2} \sigma_{n}^{2} \\
m_{Z_{n}^{Q}} & =m_{n}^{I} \bar{m}_{n}^{Q}+m_{n}^{Q} \bar{m}_{n}^{I} \\
\sigma_{Z_{n}^{Q}}^{2} & =\mu_{n}^{2} \bar{\sigma}_{n}^{2}+\bar{\mu}_{n}^{2} \sigma_{n}^{2} .
\end{aligned}
$$

Therefore, $\sqrt{\mathcal{P}} \beta_{n} Z_{n}^{I} \sim \mathcal{N}\left(\sqrt{\mathcal{P}} \beta_{n} m_{Z_{n}^{I}}, \beta_{n}^{2} \mathcal{P} \sigma_{Z_{n}}^{2}\right)$ and $\sqrt{\mathcal{P}} \beta_{n} Z_{n}^{Q} \sim \mathcal{N}\left(\beta_{n} \sqrt{\mathcal{P}} m_{Z_{n}^{Q}}, \beta_{n}^{2} \mathcal{P} \sigma_{Z_{n}}^{2}\right)$. Consequently, the PDF of $Z_{n}$ can be approximated as $Z_{n} \sim \mathcal{C N}\left(m_{Z_{n}}, 2 \sigma_{Z_{n}}^{2}\right)$, where $m_{Z_{n}}=$ $m_{Z_{n}^{I}}+j m_{Z_{n}^{Q}}$ and $\sigma_{Z_{n}}^{2}=\sigma_{Z_{n}^{I}}^{2}=\sigma_{Z_{n}^{Q}}^{2}$. In addition, as can be noted from the statistics of $Z_{n}^{I}$ and $Z_{n}^{Q}$ in (13)-(16), $Z_{n}^{I}$ and $Z_{n}^{Q}$ have the same variance but different mean values. Consequently, the distribution of $\varpi_{n}$ can be approximated by a Rician PDF with mean and variance that are given 
by

$$
\begin{gathered}
\mu_{Z_{n}}=\left|m_{Z_{n}}\right|^{2} \\
=\bar{\mu}_{n} \mu_{n} \\
\sigma_{Z_{n}}^{2}=\mu_{n}^{2} \bar{\sigma}_{n}^{2}+\bar{\mu}_{n}^{2} \sigma_{n}^{2} .
\end{gathered}
$$

Consequently, the Rician PDF parameters $\Omega_{\varpi_{n}}$ and $K_{\varpi_{n}}$ can be written as

$$
\begin{gathered}
\Omega_{\varpi_{n}}=\mu_{Z_{n}}^{2}+2 \sigma_{Z_{n}}^{2} \\
=\bar{\mu}_{n}^{2} \mu_{n}^{2}+2\left(\mu_{n}^{2} \bar{\sigma}_{n}^{2}+\bar{\mu}_{n}^{2} \sigma_{n}^{2}\right) \\
K_{\varpi_{n}}=\frac{\bar{K}_{n} K_{n}}{\bar{K}_{n}+K_{n}} .
\end{gathered}
$$

Based on (20), a cascaded channel of two Rician channels having Rician factors $\bar{K}$ and $K$ can be approximated as a Rician channel with Rician factor $K_{\varpi_{n}}<\min \left(\bar{K}_{n}, K_{n}\right)$ given that $\bar{K}_{n}$ and $K_{n}$ are large.

By simple random variable transformation, the PDF of $\lambda_{n}=\sqrt{\mathcal{P}} \beta_{n} \varpi_{n}$ can be found as Rician with the following distribution.

$$
f_{\lambda_{n}}\left(\lambda_{n}\right)=\frac{2\left(1+K_{\lambda_{n}}\right)}{\Omega_{\lambda_{n}} \mathrm{e}^{K_{\lambda_{n}}}} \lambda_{n} \mathrm{e}^{-\frac{\left(1+K_{\lambda_{n}}\right)}{\Omega_{\lambda_{n}}} \lambda_{n}^{2}} I_{0}\left(2 \lambda_{n} \sqrt{\frac{K_{\lambda_{n}}\left(1+K_{\lambda_{n}}\right)}{\Omega_{\lambda_{n}}}}\right)
$$

where $K_{\lambda_{n}}=K_{\varpi_{n}}$ and $\Omega_{\lambda_{n}}=\Omega_{\varpi_{n}} \mathcal{P} \beta_{n}^{2}$. However, the distribution of the sum of multiple Rician random variables, $\Lambda=\sum_{n} \lambda_{n}$, does not have a closed form expression. Therefore, an accurate closed-form approximation will be used as described in [61] to enable analytical performance evaluation. It is worth noting that there is a typo in [61, Eq. 5], where the first term should be $1 / \sqrt{2 \pi \sigma_{\lambda_{n}}^{2}}$ instead of $1 / \sqrt{2 \pi}$. To simplify the discussion, it is assumed that $\beta_{n}=\beta \forall n$, and a new variable is defined as $\check{P}_{0} \triangleq \beta^{2} P_{0}$ and $\check{\mathcal{P}} \triangleq \check{P}_{0} / \sigma_{w}^{2}$, to simplify the notation. Finally, by simple random variable transformation for the distribution of $\Lambda$ given in [61], the distribution of $\gamma=\Lambda^{2}$ can be provided as

$$
f_{\gamma}(\gamma)=\frac{1}{\sigma_{\lambda_{n}} \sqrt{8 \pi N \check{\mathcal{P}} \gamma}} \mathrm{e}^{-\frac{\left(\sqrt{\frac{\gamma}{\mathcal{P}}}-\mu_{\lambda_{n}} N\right)^{2}}{2 N \sigma_{\lambda_{n}}^{2}}}+f_{c}(\gamma)
$$


where $\mu_{\lambda_{n}}$ and $\sigma_{\lambda_{n}}^{2}$ are the mean and variance of $\lambda_{n}$, respectively, and $f_{c}(\gamma)$ is a correction

$$
f_{c}(\gamma)=\frac{a_{0}}{2 a_{1} \sqrt{\mathscr{\mathcal { P }} \gamma}} \Psi_{(\gamma)}\left(\Psi_{(\gamma)}^{2}-3\right) \mathrm{e}^{-\frac{1}{2} \Psi_{(\gamma)}^{2}}
$$

where $\Psi_{(\gamma)}=\left(\sqrt{\frac{\gamma}{\mathcal{P}}}-\sqrt{N} a_{2}\right) / \sqrt{N} a_{1}$, and the coefficients $a_{0}, a_{1}$ and $a_{2}$ are used to control the amplitude, spread and shift, respectively. In [61], the values of these coefficients depend on the Rician factor $K$ and the number of random variables $N$. The method for evaluating the coefficients is introduced in [61], which is based on the least squares fitting with the exact cumulative distribution function (CDF). A table is provided in [61] for certain cases, however, these coefficients can be evaluated using nonlinear curve-fitting in least-squares sense, where the exact CDF can be obtained by convolving $N$ Rician PDFs and then performing numerical integration.

\section{Symbol ERror Rate (SER) AnAlysis}

To evaluate the SER of signalling over fading channels, the general method is to model the channel as conditionally Gaussian, obtain the SER, and then to eliminate the conditioning by averaging the conditional SER over the instantaneous SNR $\gamma$. Therefore, the average SER $\bar{P}_{S}$ can be computed as

$$
\bar{P}_{S}=\int_{0}^{\infty} P_{S}(\gamma) f_{\gamma}(\gamma) d \gamma
$$

For most widely used modulation schemes such as quadrature amplitude modulation (QAM) and phase shift keying (PSK), the conditional SER for a given $\gamma$ in the presence of AWGN can be approximated as

$$
P_{S}(\gamma) \simeq A Q(\sqrt{B \gamma})
$$

where $Q(\cdot)$ is the complementary cumulative distribution function of the Gaussian distribution, and the values of $A$ and $B$ depend on the modulation scheme [53, Table 6.1, pp. 167]. The values of $A$ and $B$ can be selected such that (25) can be used to evaluate the bit error rate (BER). 
A. Single reflector $(N=1)$, single hop $(L=1)$

The SER for the single reflector case can be obtained by substituting (8) and (25) into (24), and defining $\sqrt{\gamma} \triangleq y$, which yields

$$
\bar{P}_{S}=2 A C_{n} \sum_{i=0}^{\infty} \sum_{l=0}^{\infty} D_{i, l}^{n} \int_{0}^{\infty} y^{i+l+1} Q(\sqrt{B} y) \mathcal{K}_{i-l}(E y) d y
$$

As can be $\mathrm{d}$ that closed form for the integral is very difficult to obtain. Therefore, approximating either the Q-function or the modified Bessel function can result in a solvable integration. However, to make the integration feasible, tight and tractable approximation for $Q(x)$, which has been proposed in [60], is applied. The approximation is based on a truncated series and given by

$$
Q(x) \simeq \frac{\mathrm{e}^{-\frac{x^{2}}{2}}}{1.135 \sqrt{\pi}} \sum_{i=1}^{n_{a}} \frac{(-1)^{i+1} 1.98^{i}}{i ! 2^{\frac{i+1}{2}}} x^{i-1}
$$

where the number of terms in the summation $n_{a}$ can be selected depending on the desired tightness. Substituting (27) in (26) yields

$$
\bar{P}_{S}=\frac{2 A C_{n}}{1.135 \sqrt{\pi}} \sum_{i=0}^{\infty} \sum_{l=0}^{\infty} D_{i, l}^{n} \sum_{k=1}^{n_{a}} \frac{(-1)^{k+1} 1.98^{k} B^{\frac{k-1}{2}}}{2^{\frac{k+1}{2}} k !} \int_{0}^{\infty} y^{i+l+k} \mathrm{e}^{-\frac{B y^{2}}{2}} \mathcal{K}_{i-l}\left(E_{n} y\right) d y .
$$

The integral can be solved as described in [59, eq. (2.16.8.4)], which yields the SER in (29), where $\mathcal{W}$ is the Whittaker hypergeometric function.

$$
\bar{P}_{S}=\frac{A C_{n} \mathrm{e}^{\frac{E_{n}^{2}}{4 B}}}{1.135 \sqrt{\pi} E_{n}} \sum_{i=0}^{\infty} \sum_{l=0}^{\infty} D_{i, l}^{n} \sum_{k=1}^{n_{a}} \frac{(-1)^{k+1} 1.98^{k} B^{\frac{k-1}{2}}}{2^{\frac{k+1}{2}} k !\left(\frac{B}{2}\right)^{\frac{i+l+k}{2}}} \Gamma\left(i+\frac{k+1}{2}\right) \Gamma\left(l+\frac{k+1}{2}\right) \mathcal{W}_{-\frac{i+l+k}{2}, \frac{i-l}{2}}\left(\frac{E_{n}^{2}}{2 B}\right)
$$

\section{B. Multiple reflectors $(N \geq 2)$, single hop $(L=1)$}

The average SER $\bar{P}_{S}$ for this case can be derived by substituting (25) and (22) into (24), which after some manipulations, as shown in Appendix I, gives

$$
\bar{P}_{S}=A\left(\frac{1}{\sigma_{\lambda_{n}} \sqrt{2 \pi N}} T_{1}+\frac{a_{0}}{a_{1}}\left[T_{2}-3 T_{3}\right]\right)
$$


where the integrals $T_{1}, T_{2}$ and $T_{3}$ are evaluated in Appendix I and the final solutions are given by

$$
\begin{gathered}
T_{1}=\frac{\mathrm{e}^{-\frac{\mu_{\lambda_{n} N}^{2} N}{2 \sigma_{\lambda_{n}}^{2}}}}{1.135 \sqrt{\pi}} \sum_{k=1}^{n_{a}} \frac{(-1)^{k+1} 1.98^{k}}{2^{\frac{k+1}{2}} k !}(B \check{\mathcal{P}})^{\frac{k-1}{2}} \frac{\Gamma(k)}{\rho_{n}^{k}} \mathrm{e}^{\frac{\mu_{\lambda_{n}}^{2}}{4 \rho_{n}^{2} \sigma_{\lambda_{n}}^{4}}} \mathcal{D}_{-k}\left(\frac{-\mu_{\lambda_{n}}}{\sigma_{\lambda_{n}}^{2} \rho_{n}}\right) \\
T_{2}=\sqrt{\frac{N}{2 \pi}} a_{1} \mathrm{e}^{-\frac{1}{2} \dot{a}}\left(\sqrt{\frac{\pi}{2}}(2+\dot{a})-\mathrm{e}^{\frac{\nu^{2}}{8 \delta}} \sum_{k=1}^{3} \omega_{k} \mathcal{D}_{-k}\left(\frac{\nu}{\sqrt{2 \delta}}\right)\right) \\
T_{3}=\frac{1}{2} \sqrt{\frac{N}{\pi}} a_{1} \mathrm{e}^{-\frac{1}{2} \dot{a}}\left(\sqrt{\pi}-\frac{1}{\sqrt{\delta}} \mathrm{e}^{\frac{\nu^{2}}{8 \delta}} \mathcal{D}_{-1}\left(\frac{\nu}{\sqrt{2 \delta}}\right)\right)
\end{gathered}
$$

where $\dot{a}=a_{2}^{2} / a_{1}^{2}, \rho_{n}=\sqrt{\frac{1}{\sigma_{\lambda_{n}}^{2} N}+B \check{\mathcal{P}}}, \delta=\frac{1}{2}\left(1+\frac{1}{N a_{1}^{2} B \check{\mathcal{P}}}\right), \nu=\frac{-a_{2}}{a_{1}^{2} \sqrt{N B \check{\mathcal{P}}}}, \omega_{1}=(2+a) \frac{1}{\sqrt{2 \delta}}$, $\omega_{2}=\frac{2 \nu}{2 \delta}$ and $\omega_{3}=\frac{\delta \frac{-3}{2}}{\sqrt{2} a_{1}^{2} N B \tilde{\mathcal{P}}}$, and $\mathcal{D}_{-n}(\cdot)$ is the parabolic cylindrical function.

\section{Single and multiple reflectors $(N \geq 1)$, multiple hops $(L \geq 2)$}

To extend the SER analysis for the general case where $\{N, L\} \geq 2$, we define the vector $\hat{\mathbf{s}}=\left[\hat{s}_{1}, \hat{s}_{2}, \cdots, \hat{s}_{L}\right]$, which contains the decoded symbols of each hop. Given that symbol $s_{0}$ is transmitted, the SER can be expressed as

$$
\begin{aligned}
\bar{P}_{S} & =\operatorname{Pr}\left(s_{0} \neq \hat{s}_{L}\right) \\
& =\sum_{s_{0}} \operatorname{Pr}\left(\hat{s}_{L} \neq s_{0} \mid s_{0}\right) \operatorname{Pr}\left(s_{0}\right) \\
& =\frac{1}{M} \sum_{s_{0}} \sum_{\hat{\mathbf{s}} \in \mathbb{S}} \operatorname{Pr}\left(\hat{s}_{1} \mid s_{0}\right) \prod_{l=2}^{L} \operatorname{Pr}\left(\hat{s}_{l} \mid \hat{s}_{l-1}\right)
\end{aligned}
$$

where $M$ is the modulation order, $\operatorname{Pr}\left(\hat{s}_{1} \mid s_{0}\right)$ is the conditional probability for the first hop, and $\operatorname{Pr}\left(\hat{s}_{l} \mid \hat{s}_{l-1}\right)$ is the conditional probability for the remaining $L-1$ hops. The vector $\hat{\mathbf{s}} \in \mathbb{S}$, where $\mathbb{S}$ is the set of all vectors that has $\hat{s}_{L} \neq s_{0}$. Evaluating (34) can be performed using the approach described in [67], [68], which for the special case of BPSK is given by

$$
\bar{P}_{S}=\frac{1}{2}-\frac{1}{2} \prod_{l=1}^{L}\left(1-2 \bar{P}_{S, l}\right)
$$

where $\bar{P}_{S, l}$ is the average symbol/bit error rate for the $l$ th hop. 


\section{Outage Probability}

The OP is defined as the probability that the SNR is below a certain threshold $\gamma_{\mathrm{th}}$, will be also denoted as $\chi$ for notational simplicity,

$$
\bar{P}_{O}=\int_{0}^{\chi} f_{\gamma}(\gamma) d \gamma
$$

A. Single reflector $(N=1)$, single hop $(L=1)$

By substituting (8) into (36), and applying the series expansion of the modified Bessel function, the OP can be found as

$$
\bar{P}_{O}=C_{n} \sum_{i=0}^{\infty} \sum_{l=0}^{\infty} D_{i, l}^{n} \int_{0}^{\chi} \gamma^{\frac{i+l}{2}} \mathcal{K}_{i-l}\left(E_{n} \sqrt{\gamma}\right) d \gamma
$$

By substituting $y=\gamma / \chi$, and after some simplifications $\bar{P}_{O, 1}$ can be expressed as

$$
\bar{P}_{O}=C_{n} \sum_{i=0}^{\infty} \sum_{l=0}^{\infty} D_{i, l}^{n} \chi^{\frac{i+l}{2}+1} \int_{0}^{1} y^{\frac{i+l}{2}} \mathcal{K}_{i-l}\left(E_{n} \sqrt{y \varkappa}\right) d y .
$$

Based on [62, Eq. 6.592.2], $\bar{P}_{O}$ can be solved in a closed-form in terms of the Meijer $G$ function,

$$
\bar{P}_{O}=C_{n} \sum_{i=0}^{\infty} \sum_{l=0}^{\infty} \frac{2^{i-l-1}}{E_{n}^{i-l}} D_{i, l}^{n} \chi^{l+1} G_{1,3}^{2,1}\left(\frac{\chi E_{n}^{2}}{4} \mid \begin{array}{c}
-l \\
i-l, 0,-l-1
\end{array}\right) .
$$

\section{B. Multiple reflectors $(N \geq 2)$, single hop $(L=1)$}

The OP for this case is derived by substituting (22) in (36) and evaluating the integral. The resultant expression can be written as

$$
\bar{P}_{O}=\bar{P}_{O}^{(a)}+\bar{P}_{O}^{(b)}-\bar{P}_{O}^{(c)}
$$

where the expressions of $\bar{P}_{O}^{(a)}, \bar{P}_{O}^{(b)}$ and $\bar{P}_{O}^{(c)}$ are given by

$$
\begin{aligned}
& \bar{P}_{O}^{(a)}=\frac{1}{2}\left[\operatorname{erf}\left(\frac{\mu_{\lambda_{n}} \sqrt{N}}{\sqrt{2} \sigma_{\lambda_{n}}}\right)-\operatorname{erf}\left(\frac{-\check{\chi}+\mu_{\lambda_{n}} N}{\sqrt{2 N} \sigma_{\lambda_{n}}}\right)\right] \\
& \bar{P}_{O}^{(b)}=\frac{a_{0} \mathrm{e}^{-\frac{1}{2} \dot{a}}}{a_{1}^{2} \sqrt{N}}\left\{N\left(a_{2}^{2}+2 a_{1}^{2}\right)+\Gamma_{\text {th }} \mathrm{e}^{-\frac{\check{\chi}^{2}-2 \sqrt{N} a_{2} \check{\chi}}{2 N a_{1}^{2}}}\right\}
\end{aligned}
$$




$$
\bar{P}_{O}^{(c)}=3 a_{0} \sqrt{N} \mathrm{e}^{-\frac{1}{2} \dot{a}}\left(1-\mathrm{e}^{-\check{\chi}\left(\frac{\dot{\chi}-2 \sqrt{N} a_{2}}{2 N a_{1}^{2}}\right)}\right)
$$

where $\operatorname{erf}(\cdot)$ is the error function, $\check{\chi}=\sqrt{\frac{\chi}{\mathcal{P}}}$ and $\Gamma_{\text {th }}=-N a_{2}^{2}+2 \sqrt{N} a_{2} \check{\chi}-\check{\chi}^{2}-2 N a_{1}^{2}$.

\section{Multiple reflectors $(N \geq 2)$, multiple hops $(L \geq 2)$}

Given that the signal will undergo $L$ hops, the outage event occurs if one or more of the $L$ hops go through an outage. Therefore, OP can be formulated as [63], [64],

$$
\bar{P}_{O}=1-\prod_{l=1}^{L}\left(1-\bar{P}_{O, l}\right)
$$

where $\bar{P}_{O, l}$ is the OP for the lth hop, which is given by (39) and (40) for $N=1$ and $N \geq 2$, respectively.

\section{NumericAl RESULTS}

This section presents the analytical and simulation results for OP and SER of the considered system. The simulation results are obtained using Monte Carlo simulation, where each simulation run consists of $10^{7}$ realizations. The links TxBS-IRS and IRS-RxBS are considered independent identically distributed (i.i.d.) flat Rician fading channels where $K_{n}=\bar{K}_{n}=K$, unless mentioned otherwise. The average transmission power of the TxBS $P_{0}$ and the reflection coefficient $\beta$ are normalized to unity, i.e., $\beta=P_{0}=1$, and the SNR is defined as $\mathcal{P}=P_{0} / \sigma_{w}^{2}$.

Figs. 2 and 3 show the SER for the single hop case, $L=1$, using various values of $N$ using BPSK and QPSK, respectively, and using $K=10 \mathrm{~dB}$. As can be noted from the two figures, the analytical and simulation results for the single reflector case, $N=1$, match very well for the considered range of SNR because the cascaded channel PDF is exact, and the only approximation used is for the Q-function. For the multiple reflectors case, the PDF approximation of the cascaded channel resulted in some discrepancies for the $N=2,3$ cases, at high SNRs. Such results are obtained because the approximation error is relatively more significant for $N<4$, particularly at high SNRs where the effect of the AWGN is small when compared to the approximation error. As can be noted from the figures, increasing the number of reflectors $N$ effectively improves the SNR, or equivalently, it enhances the SER considerably. Nevertheless, the improvement gained 


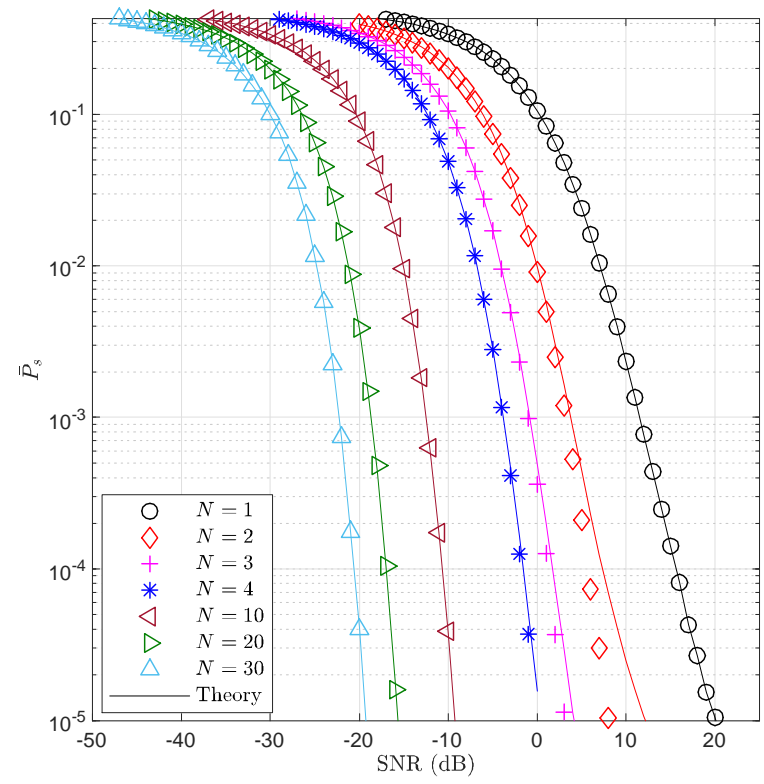

Fig. 2. SER using BPSK for various values of $N, K=10 \mathrm{~dB}$ and $L=1$.

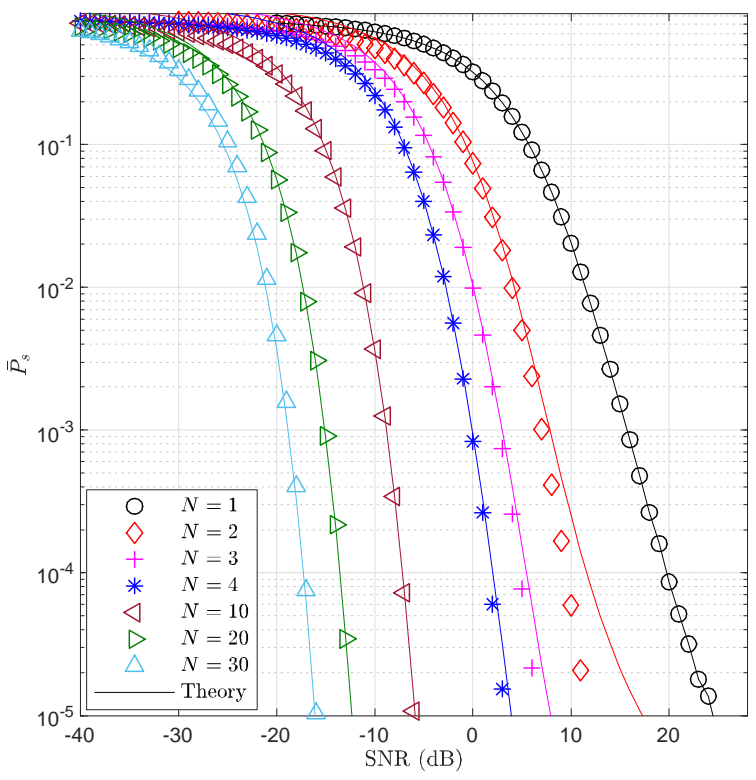

Fig. 3. SER using QPSK using various values of $N, K=10 \mathrm{~dB}$ and $L=1$. 


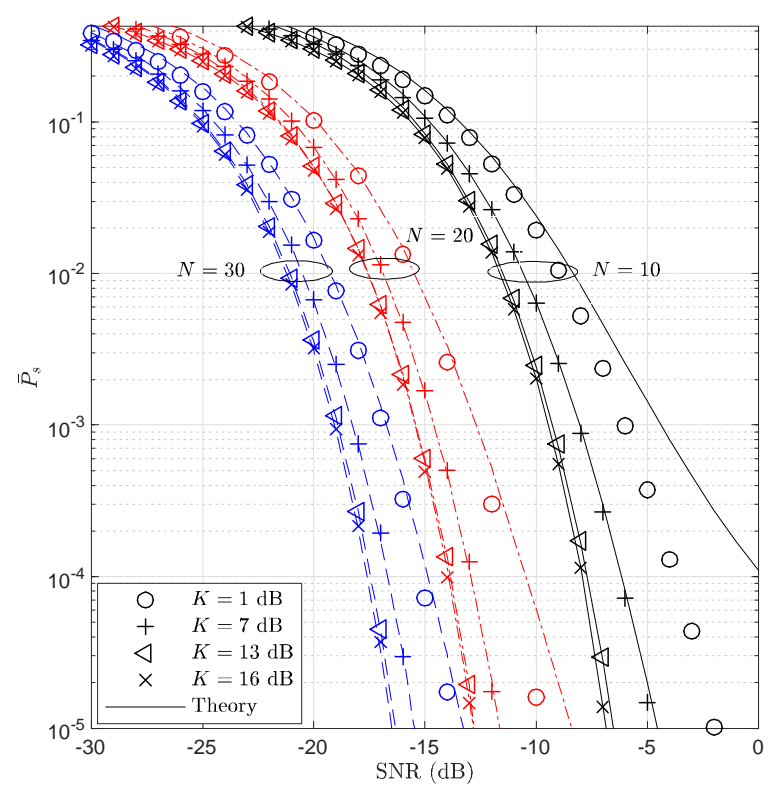

Fig. 4. SER using QPSK for various values of $N$ and $K, L=1$.

by increasing $N$ decreases for large values of $N$. For example, the gain obtained using $N=2$ as compared to the case of $N=1$ is about $8 \mathrm{~dB}$ at $\bar{P}_{S}=10^{-4}$, increasing $N$ from 2 to 3 provides only and additional $6 \mathrm{~dB}$, and so forth. Interestingly, increasing $N$ to 30 provides about $36 \mathrm{~dB}$ of SNR improvement. The significant SNR improvement can be exploited to increase the modulation order, and thus enhance the spectral efficiency.

Fig. 4 shows the analytical and simulated SER versus SNR using QPSK for various values of $N$ and $K$. As can be noted from the figure, the derived approximation matches the simulation results very well for high values of $K$ for the considered SNR range. For small $K$ values, the difference between the approximated and simulation results is noticeable for $N=10$, and it decreases by increasing $N$. It can be also noted that the approximation error becomes less significant at low SNRs because the performance in such regions is dominated by the AWGN. The impact of the channel quality on SER decreases by increasing $N$, which is demonstrated by the condensed SER curves for large values of $N$.

Fig. 5 presents the analytical and simulated SER using BPSK for various values of $N$ and $L$, where the transmitted power per each TxBS and the Rician factor $K$ are fixed at 0 and $10 \mathrm{~dB}$, respectively. The results show the high accuracy of the approximations used because $K \gg 1 \mathrm{~dB}$. 


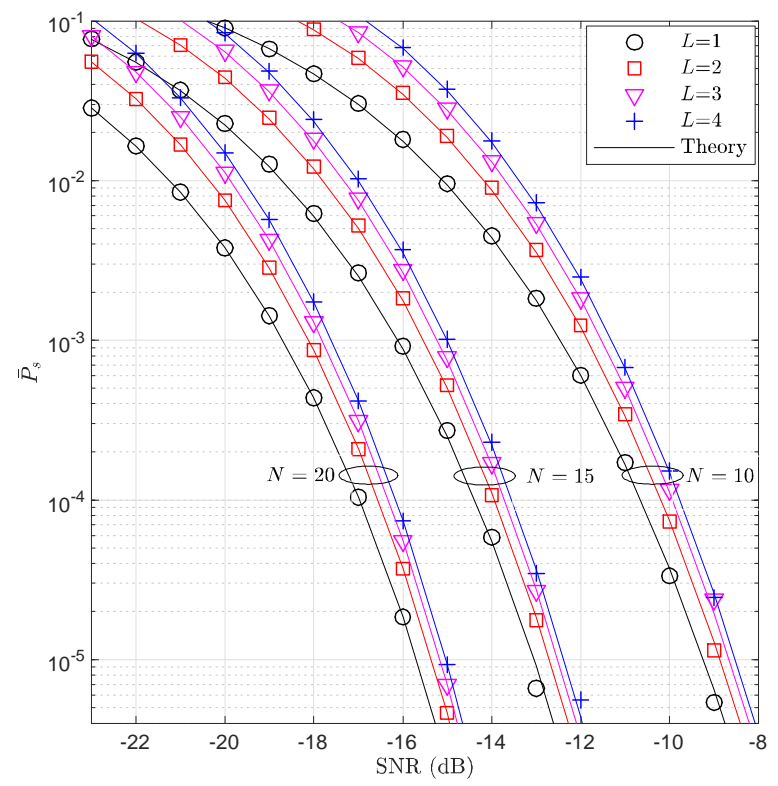

Fig. 5. SER using BPSK for varaious values of $L$ and $N, K=10 \mathrm{~dB}$.

According to the figure, the main difference between the IRS and a conventional multihop system in Rayleigh fading channels is that the SER degradation obtained by increasing $L$ from 1 to 2 is about $0.35 \mathrm{~dB}$ at $10^{-5}$, while it is about $3 \mathrm{~dB}$ for a conventional multihop system. Increasing the value of $L$ beyond 4 has very limited effect on the SER.

Fig. 6 presents the results of the OP for various values of $\gamma_{\text {th }}$, i.e., $\chi$, and $N$. The Rician factor $K=10 \mathrm{~dB}, \gamma_{\mathrm{th}}=[5,10,15] \mathrm{dB}$, and $N=[10,20]$. As the figure shows, derived OP shows an excellent match with simulation results for all of the considered scenarios. Clearly, increasing $\gamma_{\text {th }}$ for a given value of $N$ deteriorates $\mathrm{OP}$, however, the degradation can be compensated by increasing $N$. It is interesting to note that increasing $\gamma_{\text {th }}$ by a certain value degrades $\bar{P}_{O}$ approximately by the same value. For example, when $N=10$, increasing $\gamma_{\text {th }}$ from 5 to $10 \mathrm{~dB}$ increases the SNR required to achieve $\bar{P}_{O}=10^{-5}$ from -5 to $-10 \mathrm{~dB}$.

Fig. 7 shows the OP versus SNR for various values of $K$, where $N=10, L=1$, and $\gamma_{\text {th }}=10$ dB. As shown in the figure, the difference between the analytical and simulation results is about $0.3 \mathrm{~dB}$ at $\bar{P}_{O}=10^{-4}$, it decreases by decreasing SNR. The approximation becomes more accurate for larger values of $K$. The figure also shows the impact of $K$ on $\bar{P}_{O}$, where increasing $K$ can significantly improve $\bar{P}_{O}$, particularly when $K$ has originally small values. For example, the SNR 


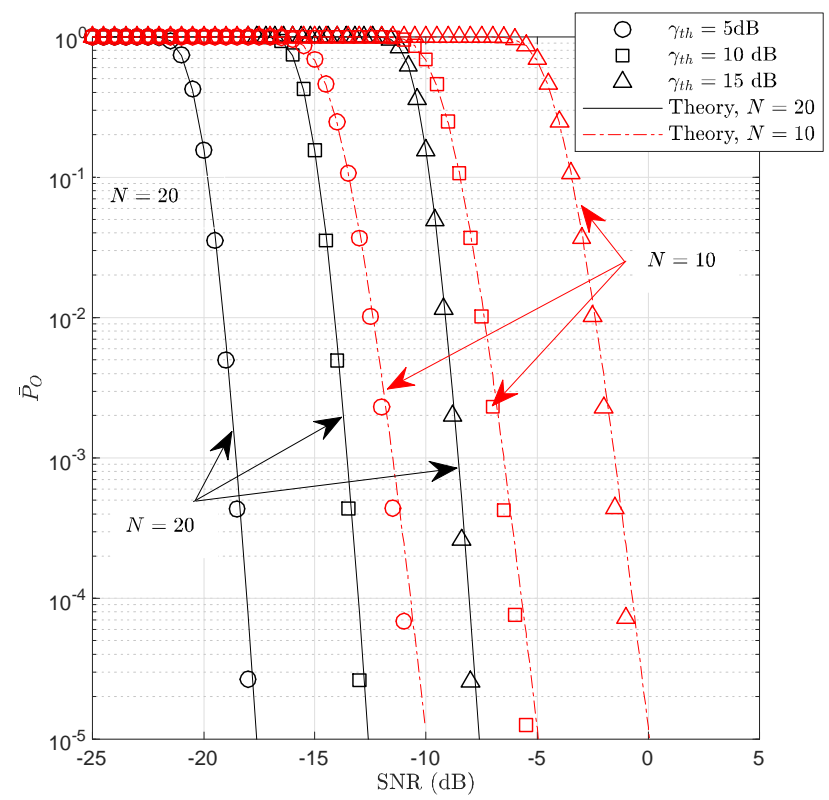

Fig. 6. The OP for various values of $N$ and $\gamma_{\mathrm{th}}, K=10 \mathrm{~dB}$ and $L=1$.

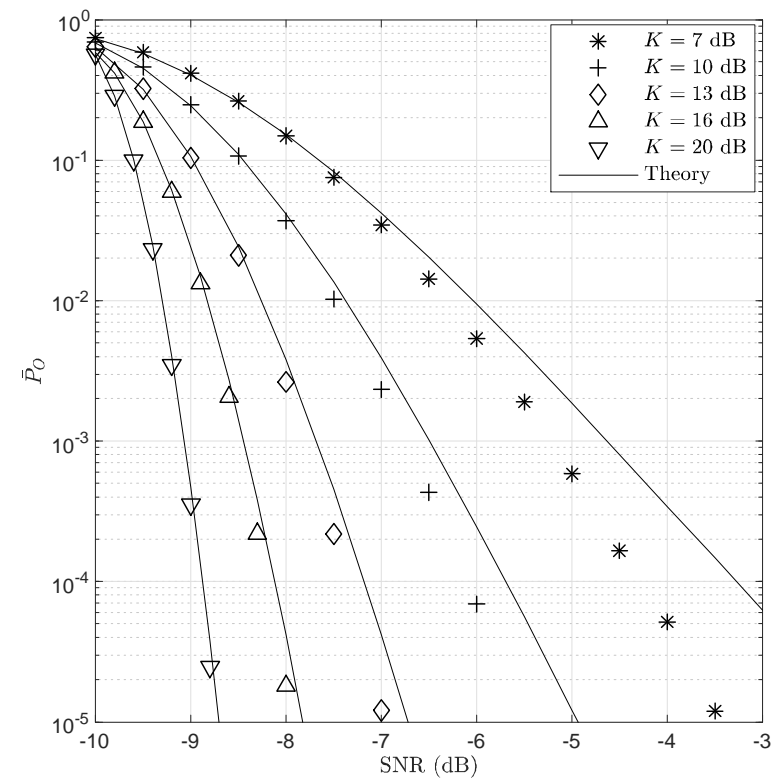

Fig. 7. OP for various values of $K$, where $N=10, \gamma_{\text {th }}=10 \mathrm{~dB}$ and $L=1$. 


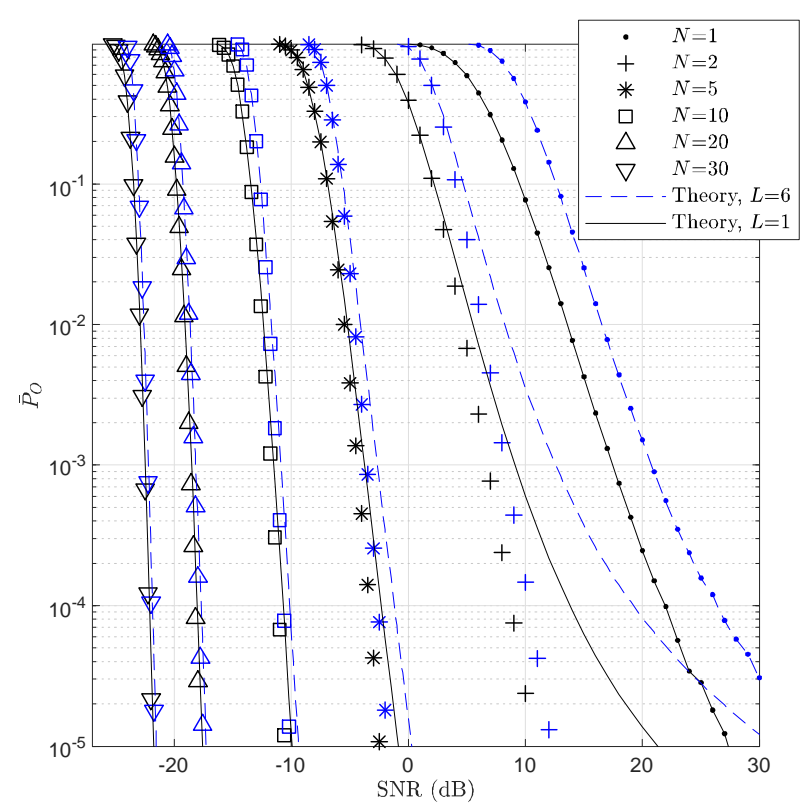

Fig. 8. OP for various values of $N, L=1$ and $6, K=10 \mathrm{~dB}$ and $\gamma_{\text {th }}=5 \mathrm{~dB}$.

required to obtain $P_{O}=10^{-5}$ can be reduced by about $2.5 \mathrm{~dB}$ when $K$ increases from $4 \mathrm{~dB}$ to $7 \mathrm{~dB}$, while the gain is reduced to less than $1 \mathrm{~dB}$ when $K$ increases from $16 \mathrm{~dB}$ to $20 \mathrm{~dB}$.

Fig. 8 presents the analytical and simulated $\mathrm{OP}$ for various values of $L$ and $N, K=10$ $\mathrm{dB}$ and $\gamma_{\mathrm{th}}=5 \mathrm{~dB}$. As can be noticed from the figure, the analytical and simulation results match very well for $N=1$ because $\bar{P}_{O}$ is exact for this case, and for $N \geq 5$ because the approximation accuracy improves versus $N$. Moreover, it can be noted that increasing $N$ improves $\bar{P}_{O}$ significantly, and dilutes the degradation caused by increasing $L$. More specifically, the effect of $N$ becomes negligible for $N \geq 10$.

\section{CONCLUSION AND FUTURE WORK}

This paper have investigated the performance of the promising IRS technology for wireless mesh backhauling, where data traffics for each BS reaches the corenetwork through multiple wireless hops. The proposed system model was analyzed for single hop, and then generalized to multiple hops. The system performance was evaluated in terms of SNR, SER and OP were exact and approximated analytical expressions were derived for IRS systems over Rician fading channels. The analytical results were verified using Mote Carlo simulation, and the extensive comparisons 
confirmed the accuracy of the derived solutions, particularly when the number of reflectors $N$ and the Rician $K$ factors are high. In addition, the results showed that the proposed IRS based backhauling can be considered as an attractive solution for wireless backhauling because it can boost the effective SNR, and reduce OP and SER considerably. Consequently, IRS is an energy and spectrum efficiency enabler for wireless backhauling.

Our Future work will focus on investigating the IRS backhauling system in multihop scenarios where the channel estimation and compensation at each hop is imperfect, and the limitations for the timing alignment and its impact on the bandwidth will be evaluated. Moreover, the use of multi IRS between adjacent BSs will be considered.

\section{ApPEndix I: Evaluating $T_{1}, T_{2}$ AND $T_{3}$}

The SER for $N \geq 2$ is obtained by substituting (23) in (22), and then substituting the result and (25) in (24). After some mathematical manipulations, the resulting integral can be expressed in the form of (30) with $T_{1}, T_{2}$ and $T_{3}$ given by

$$
\begin{aligned}
& T_{1}=\frac{1}{2 \sqrt{\check{\mathcal{P}}}} \int_{0}^{\infty} \frac{Q(\sqrt{B \gamma})}{\sqrt{\gamma}} \mathrm{e}^{-\frac{\left(\sqrt{\frac{\gamma}{\mathcal{P}}}-\mu_{\lambda_{n}} N\right)^{2}}{2 N \sigma_{\lambda_{n}}^{2}}} d \gamma \\
& T_{2}=\frac{1}{2 \sqrt{\check{\mathcal{P}}}} \int_{0}^{\infty} \frac{\Psi_{(\gamma)}^{3}}{\sqrt{\gamma}} Q(\sqrt{B \gamma}) \mathrm{e}^{-\frac{1}{2} \Psi_{(\gamma)}^{2}} d \gamma \\
& T_{3}=\frac{1}{2 \sqrt{\mathcal{P}}} \int_{0}^{\infty} Q(\sqrt{B \gamma}) \frac{\Psi_{(\gamma)}}{\sqrt{\gamma}} \mathrm{e}^{-\frac{1}{2} \Psi_{(\gamma)}^{2}} d \gamma
\end{aligned}
$$

These three integrals are solved in the three subsections below.

\section{A. Evaluating the Integral $T_{1}$}

By substituting $y=\sqrt{\frac{\gamma}{\mathcal{P}}}$, the integral $T_{1}$ in (45) is reduced to

$$
T_{1}=\int_{0}^{\infty} Q(\sqrt{B \check{\mathcal{P}}} y) \mathrm{e}^{-\frac{1}{2 N}\left(\frac{y-N \mu_{\lambda_{n}}}{\sigma_{\lambda_{n}}}\right)^{2}} d y
$$


The approximation of the Q-function provided in (27) is applied, and then the resulting integral can be written as

$$
T_{1}=\frac{\mathrm{e}^{-\frac{\mu_{\lambda_{n}}^{2} N}{2 \sigma_{\lambda_{n}}^{2}}}}{1.135 \sqrt{\pi}} \sum_{i=1}^{n_{a}} \frac{(-1)^{i+1} 1.98^{i}}{2^{\frac{i+1}{2}} i !}(B \check{\mathcal{P}})^{\frac{i-1}{2}} \int_{0}^{\infty} y^{i-1} \mathrm{e}^{-\frac{1}{2}\left(\frac{1}{N \sigma_{\lambda_{n}}^{2}}+B \check{\mathcal{P}}\right) y^{2}+\frac{\mu_{\lambda_{n}}}{\sigma_{\lambda_{n}}^{2}} y} d y
$$

which can be solved as $[62,3.462 .1]$

$$
T_{1}=\frac{\mathrm{e}^{-\frac{\mu_{\lambda_{n}}^{2} N}{2 \sigma_{\lambda_{n}}^{2}}}}{1.135 \sqrt{\pi}} \sum_{i=1}^{n_{a}} \frac{(-1)^{i+1} 1.98^{i}}{2^{\frac{(i+1)}{2}} i !}(B \check{\mathcal{P}})^{\frac{i-1}{2}} \frac{\Gamma(i)}{\rho_{n}^{i}} \mathrm{e}^{\frac{\mu_{\lambda_{n}}^{2}}{4 \rho_{n}^{2} \sigma_{\lambda_{n}}^{4}}} \mathcal{D}_{-i}\left(\frac{-\mu_{\lambda_{n}}}{\sigma_{\lambda_{n}}^{2} \rho_{n}}\right)
$$

where $\rho_{n}=\sqrt{\frac{1}{\sigma_{\lambda_{n}}^{2} N}+B \check{\mathcal{P}}}, \Gamma(\cdot)$ is the Gamma function, and $\mathcal{D}_{-n}(\cdot)$ is the parabolic cylinder function.

\section{B. Evaluating the Integral $T_{2}$}

By substituting $x=\sqrt{\gamma}$, the integral $T_{2}$ in (46) is reduced to

$$
T_{2}=\frac{1}{\sqrt{\mathscr{P}}} \int_{0}^{\infty} \Psi_{\left(x^{2}\right)}^{3} \mathrm{e}^{-\frac{1}{2} \Psi_{\left(x^{2}\right)}^{2}} Q(\sqrt{B} x) d x
$$

The definition of the Q-function is then substituted in (51), i.e., $Q(x) \triangleq \frac{1}{\sqrt{2 \pi}} \int_{x}^{\infty} \mathrm{e}^{-\frac{y^{2}}{2}} d y$, and subsequently, the order of the resulting double integration is changed. Consequently, $T_{2}$ can be written as

$$
T_{2}=\frac{1}{\sqrt{2 \pi \check{\mathcal{P}}}} \int_{0}^{\infty} \mathrm{e}^{-\frac{y^{2}}{2}} T_{2,1} d y
$$

where $T_{2,1}$ is given by

$$
T_{2,1}=\int_{0}^{\frac{y}{\sqrt{B}}} \Psi_{(x)}^{3} \mathrm{e}^{-\frac{1}{2} \Psi_{(x)}^{2}} d x
$$

Therefore, $T_{2,1}$ should be solved first, then the result is substituted in (52) to solve $T_{2}$. By the change of variables $\Psi_{\left(x^{2}\right)}=\left(\sqrt{\frac{x^{2}}{\mathcal{P}}}-\sqrt{N} a_{2}\right) /\left(\sqrt{N} a_{1}\right)$ in (53), $T_{2,1}$ can be written as

$$
T_{2,1}=\sqrt{N \check{\mathcal{P}}} a_{1} \int_{\frac{-a_{2}}{a_{1}}}^{\Psi_{\left(y^{2} / B\right)}^{2}} \Psi_{(x)}^{3} \mathrm{e}^{-\frac{1}{2} \Psi_{\left(x^{2}\right)}^{2}} d \Psi_{\left(x^{2}\right)}
$$


which can be solved as [62]

$$
T_{2,1}=\sqrt{N \check{\mathcal{P}}} a_{1}\left[(2+\dot{a}) \mathrm{e}^{-\frac{1}{2} \dot{a}}\left(2+\Psi_{\left(\frac{y^{2}}{B}\right)}^{2}\right)-\mathrm{e}^{-\frac{1}{2} \Psi^{2}\left(\frac{y^{2}}{B}\right)}\right]
$$

Consequently, (??) is substituted in (52), $T_{2}$ is reduced to

$$
T_{2}=\sqrt{\frac{N}{2 \pi}} a_{1}\left[(2+a ́) \mathrm{e}^{-\frac{1}{2} a} \int_{0}^{\infty} \mathrm{e}^{-\frac{y^{2}}{2}} d y-\int_{0}^{\infty}\left(2+\Psi_{\left(\frac{y^{2}}{B}\right)}^{2}\right) \mathrm{e}^{-\frac{y^{2}}{2}-\frac{1}{2} \Psi^{2}}\left(\frac{y^{2}}{B}\right) d y\right]
$$

By using the definition of the Q-function, it can be easily shown that $\int_{0}^{\infty} \mathrm{e}^{-\frac{y^{2}}{2}}=\sqrt{\frac{\pi}{2}}$. After some algebraic operations, $T_{2}$ can be simplified to

$$
\begin{aligned}
T_{2}=\sqrt{\frac{N}{2 \pi}} a_{1} \mathrm{e}^{-\frac{1}{2} a}\left[\sqrt{\frac{\pi}{2}}(2+\dot{a})\right. & -(2+\dot{a}) \int_{0}^{\infty} \mathrm{e}^{\left[-\delta y^{2}-\nu y\right]} d y \\
& \left.-2 \nu \int_{0}^{\infty} y \mathrm{e}^{\left[-\delta y^{2}-\nu y\right]} d y-\frac{1}{N a_{1}^{2} B \check{\mathcal{P}}} \int_{0}^{\infty} y^{2} \mathrm{e}^{\left[-\delta y^{2}-\nu y\right]} d y\right]
\end{aligned}
$$

Thereafter, the integration can be evaluated in closed form as [62, 3.462.1]

$$
T_{2}=\sqrt{\frac{N}{2 \pi}} a_{1} \mathrm{e}^{-\frac{1}{2} \dot{a}}\left[\frac{\sqrt{\pi}}{(2+\hat{a})^{-1}}-\mathrm{e}^{\frac{\nu^{2}}{8 \delta}}\left[\frac{(2+a ́)}{\sqrt{\delta}} \mathcal{D}_{-1}\left(\frac{\nu}{\sqrt{2 \delta}}\right)+\frac{\nu \sqrt{2}}{\delta} \mathcal{D}_{-2}\left(\frac{\nu}{\sqrt{2 \delta}}\right)+\frac{\delta^{\frac{-3}{2}}}{N a_{1}^{2} B \check{\mathcal{P}}} \mathcal{D}_{-3}\left(\frac{\nu}{\sqrt{2 \delta}}\right)\right]\right]_{(58)}
$$

Finally, the expression in (??) can be written using the summation notation, where the result is expressed in (32) where $\delta$ and $\nu$ are defined below (33).

\section{Evaluating the Integral $T_{3}$}

The steps followed to evaluate $T_{3}$ is very quiet similar to the procedure used to evaluate $T_{2}$, and therefore the derivation of $T_{3}$ is not included for the sake of brevity.

\section{REFERENCES}

[1] Ericsson Mobility Report 2019. Accessed: June 15, 2020. [Online]. Available: https://www.ericsson.com/4acd7e/assets/local/mobility-report/documents/2019/emr-november-2019.pdf.

[2] Cisco. 2020 Cisco annual internet report. [Online]. Available: https://www.cisco.com/c/en/us/solutions/ collateral/executiveperspectives/annual-internet-report/white-paper-c11-741490.html.

[3] J. Rygaard, “Digitization, densification and why you should care,” Nokia, Tech. Rep., Feb. 2017. [Online]. Available: https: www.nokia.com/blog/digitization-densification-care/ 
[4] M. Mirahmadi, A. Al-Dweik, and A. Shami, "Interference modeling and performance evaluation of heterogeneous cellular networks," IEEE Trans. Commun., vol. 62, no. 6, pp. 2132-2144, June 2014.

[5] J. G. Andrews, H. Claussen, M. Dohler, S. Rangan, and M. C. Reed, "Femtocells: Past, present, and future," IEEE J. Sel. Areas Commun., vol. 30, no. 3, pp. 497-508, 2012.

[6] RSPG Report on Spectrum Issues on Wireless Backhaul, Radio Spectrum Policy Group, European Commission, DirectorateGeneral for Communications Networks, Content and Technology, RSPG15-607, June 2015.

[7] W. Abderrahim, O. Amin, M. Alouini and B. Shihada, "Latency-aware offloading in integrated satellite terrestrial networks," IEEE Open J. Commun. Soc., vol. 1, pp. 490-500, 2020.

[8] Y. Liu, A. Tang and X. Wang, "Joint incentive and resource allocation design for user provided network under 5G integrated access and backhaul networks," IEEE Trans. Netw. Sci. Eng., vol. 7, no. 2, pp. 673-685, 1 Apr. 2020.

[9] C. Saha and H. S. Dhillon, "Millimeter wave integrated access and backhaul in 5G: performance analysis and design insights," IEEE J. Sel. Areas Commun., vol. 37, no. 12, pp. 2669-2684, Dec. 2019.

[10] N. Kim and D. Cho, "A novel synchronous duplexing method for long distance wireless backhaul system," IEEE Wireless Commun. Lett., vol. 8, no. 3, pp. 761-764, Jun. 2019.

[11] “Terragraph virtual fiber for high speed fixed broadband," Telecom infra project, White Paper, 2018. [Online]. Available: https://terragraph.com/wp-content/uploads/2019/02/Terragraph_MikebudaTrial_Whitepaper.pdf

[12] M. Coldrey, "Maturity and field proven experience of millimetre wave transmission," Sophia Antipolis, France, ETSI White Paper, Sep. 2015. [Online]. Available: https://www.etsi.org/images/files/ETSIWhitePapers/etsi_wp10_field_pr- oven_experience_of_mwt_20150923.pdf

[13] “Telecom infra project mmWave radio link aspects," Telecom infra project, White Paper, 2018. [Online]. Available: https://telecominfraproject.com/wp-content/uploads/TIP-mmWave_Radio-Link-Aspects_October-2018_vF.pdf

[14] “Telecom infra project customer premises equipment (CPE) market adoption requirements," Telecom infra project, White paper, 2018. [Online]. Available: https://telecominfraproject.com/wp-content/uploads/TIP-mmWave-CPE-Market-AdoptionRequirements_October-2018_vF.pdf

[15] O. Tipmongkolsilp, S. Zaghloul and A. Jukan, "The evolution of cellular backhaul technologies: Current issues and future trends," IEEE Commun. Surveys Tuts., vol. 13, no. 1, pp. 97-113, First Quarter 2011.

[16] K. Zheng, L. Zhao, J. Mei, M. Dohler, W. Xiang, and Y. Peng, "10 Gb/s hetsnets with millimeter-wave communications: Access and networking - challenges and protocols," IEEE Commun. Mag., vol. 53, no. 1, pp. 222-231, Jan. 2015.

[17] R. Favraud, C. Chang, and N. Nikaein, "Autonomous self-backhauled LTE mesh network with QoS guarantee," IEEE Access, vol. 6, pp. 4083-4117, 2018.

[18] Y. Yu, T. Hsieh, and A. Pang, "Millimeter-wave backhaul traffic minimization for CoMP over 5G cellular networks," IEEE Trans. Veh. Technol., vol. 68, no. 4, pp. 4003-4015, Apr. 2019.

[19] M. Alzenad, M. Shakir, H. Yanikomeroglu, and M. Alouini, "FSO-based vertical backhaul/fronthaul framework for 5G+ wireless networks," IEEE Commun. Mag., vol. 56, no. 1, pp. 218-224, Jan. 2018.

[20] S. Samarakoon, M. Bennis, W. Saad, and M. Latva-aho, "Backhaul-aware interference management in the uplink of wireless small cell networks," IEEE Trans. Wireless Commun., vol. 12, no. 11, pp. 5813-5825, Nov. 2013.

[21] L. Sanguinetti, A. Moustakas, and M. Debbah, "Interference management in 5G reverse TDD HetNets with wireless backhaul: A large system analysis," IEEE J. Sel. Areas Commun., vol. 33, no. 6, pp. 1187-1200, Jun. 2015. 
[22] Q.-U.-A Nadeem, A. Kammoun, M. Debbah, and M. Alouini, "3D massive MIMO systems: Modeling and performance analysis," IEEE Trans. Wireless Commun., vol. 14, no. 12, pp. 6926-6939, Dec. 2015.

[23] H. Tabassum, A. Sakr, and E. Hossain, "Analysis of massive MIMO-enabled downlink wireless backhauling for full-duplex small cells," IEEE Trans. Commun., vol. 64, no. 6, pp. 2354-2369, Jun. 2016.

[24] M. Feng, S. Mao, and T. Jiang, "Joint frame design, resource allocation and user association for massive MIMO heterogeneous networks with wireless backhaul," IEEE Trans. Wireless Commun., vol. 17, no. 3, pp. 1937-1950, Mar. 2018.

[25] P. Anokye, R. Ahiadormey, C. Song, and K. Lee, "Achievable sum-rate analysis of massive MIMO full-duplex wireless backhaul links in heterogeneous cellular networks," IEEE Access, vol. 6, pp. 23456-23469, 2018.

[26] A. Bonfante et al., "5G massive MIMO architectures: self-backhauled small cells versus direct access," IEEE Trans. Veh. Technol., vol. 68, no. 10, pp. 10003-10017, Oct. 2019.

[27] E. Basar, M. Di Renzo, J. De Rosny, M. Debbah, M.-S. Alouini and R. Zhang, "Wireless communications through reconfigurable intelligent surfaces," IEEE Access, vol. 7, pp. 116753-116773, 2019.

[28] M. Di Renzo et al., "Smart radio environments empowered by reconfigurable AI meta-surfaces: An idea whose time has come," EURASIP J. Wireless Commun. Netw., vol. 2019, p. 129, May 2019.

[29] C. Huang, A. Zappone, G. Alexandropoulos, M. Debbah, and C. Yuen, "Reconfigurable intelligent surfaces for energy efficiency in wireless communication,” IEEE Trans. Wireless Commun., vol. 18, no. 8, pp. 4157-4170, Aug. 2019.

[30] Q. Wu and R. Zhang, "Intelligent reflecting surface enhanced wireless network via joint active and passive beamforming," IEEE Trans. Wireless Commun., vol. 18, no. 11, pp. 5394-5409, Nov. 2019.

[31] E. Basar, "Reconfigurable intelligent surface-based index modulation: A new beyond MIMO paradigm for 6G," IEEE Trans. Commun., IEEE Early Access. doi: 10.1109/TCOMM.2020.2971486

[32] A. Canbilen, E. Basar, and S. Ikki, "Reconfigurable intelligent surface-assisted space shift keying," 2020. [Online]. Available: https://arxiv.org/abs/2001.11287.

[33] J. Ye, S. Guo, and M.-S. Alouini, "Joint reflecting and precoding designs for SER minimization in reconfigurable intelligent surfaces assisted MIMO systems," to appear, IEEE Trans. Wireless Commun. 2020. [Online]. Available: https://arxiv.org/abs/1906.11466.

[34] D. Mishra and H. Johansson, "Channel estimation and low-complexity beamforming design for passive intelligent surface assisted MISO wireless energy transfer, ”IEEE Int. Conf. Acoustics, Speech and Signal Process. (ICASSP), Brighton, United Kingdom, 2019, pp. 4659-4663.

[35] Z. He and X. Yuan, "Cascaded channel estimation for large intelligent metasurface assisted massive MIMO," IEEE Wireless Commun. Lett., vol. 9, no. 2, pp. 210-214, Feb. 2020.

[36] Q.-U.-A. Nadeem, A. Kammoun, A. Chaaban, M. Debbah, M.-S. Alouini, "Intelligent reflecting surface assisted wireless communication: Modeling and channel estimation,” to appear, IEEE Open J. Commun. Soc. 2020. [Online]. Available: https://arxiv.org/abs/1906.02360.

[37] Q. U. Nadeem, A. Kammoun, A. Chaaban, M. Debbah, and M. -S. Alouini, "Asymptotic max-min SINR analysis of reconfigurable intelligent surface assisted MISO systems," to appear, IEEE Trans. Wireless Commun., 2020.

[38] E. Calvanese Strinati et al., "6G: The next frontier: From holographic messaging to artificial intelligence using subterahertz and visible light communication,” IEEE Veh. Technol. Mag., vol. 14, no. 3, pp. 42-50, Sep. 2019. 
[39] X. Ge, S. Tu, G. Mao, C. Wang, and T. Han, "5G ultra-dense cellular networks,” IEEE Wireless Commun., vol. 23, no. 1, pp. 72-79, Feb. 2016.

[40] R. Mesleh, H. Haas, S. Sinanovic, C. Ahn, and S. Yun, "Spatial modulation," IEEE Trans. Veh. Technol., vol. 57, no. 4, pp. 2228-2241, Jul. 2008.

[41] J. Jeganathan, A. Ghrayeb, L. Szczecinski, and A. Ceron, "Space shift keying modulation for MIMO channels," IEEE Trans. Wireless Commun., vol. 8, no. 7, pp. 3692-3703, July 2009.

[42] M. Di Renzo, H. Haas, A. Ghrayeb, S. Sugiura, and L. Hanzo, "Spatial modulation for generalized MIMO: Challenges, opportunities, and implementation," Proc. IEEE, vol. 102, no. 1, pp. 56-103, Jan. 2014.

[43] P. Liu and A. Springer, "Space shift keying for LOS communication at mmWave frequencies," IEEE Wireless Commun. Lett., vol. 4, no. 2, pp. 121-124, April 2015.

[44] M. Alshawaqfeh and R. Mesleh, "Reduced complexity sparse recovery detectors for differential space shift keying MIMO system," IEEE Trans. Veh. Technol., IEEE Early Access, 2020. doi: 10.1109/TVT.2020.2975664

[45] E. Panayirci, A. Yesilkaya, T. Cogalan, H. Poor, and H. Haas, "Physical-layer security with optical generalized space shift keying," IEEE Trans. Commun., IEEE Early Access, 2020. doi: 10.1109/TCOMM.2020.2969867

[46] Z. Ding and H. V. Poor, “A simple design of IRS-NOMA transmission,” IEEE Commun. Lett., IEEE Early Access, doi: 10.1109/LCOMM.2020.2974196

[47] Ö. Özdogan, E. Björnson, and E. Larsson, "Intelligent reflecting surfaces: Physics, propagation, and pathloss modeling," IEEE Wireless Commun. Lett., IEEE Early Access. doi: 10.1109/LWC.2019.2960779

[48] L. Dai et al., "Reconfigurable intelligent surface-based wireless communication: Antenna design, prototyping and experimental results,” 2019. [Online]. Available: https://arxiv.org/abs/1912.03620.

[49] M. Al-Jarrah, K. Park, A. Al-Dweik, and M. Alouini, "Error rate analysis of amplitude-coherent detection over Rician fading channels with receiver diversity," IEEE Trans. Wireless Commun., vol. 19, no. 1, pp. 134-147, Jan. 2020.

[50] N. O'Donoughue and J. Moura, "On the product of independent complex Gaussians," IEEE Trans. Signal Process., vol. 60, no. 3, pp. 1050-1063, March 2012.

[51] A. Seijas-Macias and A. Oliveira. "An approach to distribution of the product of two normal variables," Discussiones Mathematicae Probability and Statistics, vol. 32, no. 1-2, pp. 87-99, 2012.

[52] A Saci, A Al-Dweik, and A Shami, "Direct data detection of OFDM signals over wireless channels," 2019. [Online]. Available: https://arxiv.org/abs/1702.02366.

[53] A. Goldsmith, Wireless Communications. Cambridge University Press, 2005.

[54] J. Proakis, Digital Communications. 3rd Ed. New York: McGraw-Hill, 1995.

[55] G. Durgin, Space-Time Wireless Channels. Englewood Cliffs, NJ: Prentice Hall, 2002.

[56] R. Dana, "Statistics of sampled Rician fading," DNA-TR-92-98 Tech. Rep., Feb. 1993.

[57] M. Samimi, G. MacCartney, S. Sun and T. S. Rappaport, " 28 GHz millimeter-wave ultrawideband small-scale fading models in wireless channels," IEEE 83rd Veh. Technol. Conf.e (VTC Spring), Nanjing, 2016, pp. 1-6.

[58] M. Abramowitz and I. Stegun, Handbook of Mathematical Functions: With Formulas, Graphs, and Mathematical Tables. Dover Publications, Inc., New York, 1970.

[59] A. P. Prudnikov, Yu. A. Brychkov, and O. I. Marichev, Integrals and Series, vol. 2 Special Functions. Gordon and Breach Science Publishers, UK, 3rd edition, 1986. 
[60] Y. Isukapalli and B. Rao, “An analytically tractable approximation for the Gaussian Q-function,” IEEE Commun. Lett., vol. 12, no. 9, pp. 669-671, Sep. 2008.

[61] J. A. Lopez-Salcedo, "Simple closed-form approximation to Ricean sum distributions," IEEE Signal Process. Lett., vol. 16, no. 3, pp. 153-155, Mar. 2009.

[62] I. Ryzhik and I. Gradshteyn, Table of Integrals, Series and Products, 7th Ed. San Diego, CA, USA: Academic Press, 2007.

[63] O. Badarneh and F. Almehmadi, "Performance of multihop wireless networks in $\alpha-\mu$ fading channels perturbed by an additive generalized gaussian noise,” IEEE Commun. Lett., vol. 20, no. 5, pp. 986-989, May 2016.

[64] D. Dixit and P. Sahu, "Exact closed-form ABER for multi-hop regenerative relay systems over $\kappa$ - $\mu$ fading," IEEE Wireless Commun. Lett., vol. 6, no. 2, pp. 246-249, Apr. 2017.

[65] K. Hammad, "Radio resource management optimization for next generation wireless networks,", PhD Thesis, Western University, London, ON, Canada, 2016.

[66] M. K. Simon, "Probability Distributions Involving Gaussian Random Variables: A Handbook for Engineers and Scientists "Norwell, MA: Kluwer Academic, 2002.

[67] K. Dhaka, R. K. Mallik and R. Schober, "Performance analysis of decode-and-forward multi-hop communication: A difference equation approach,” IEEE Trans. Commun., vol. 60, no. 2, pp. 339-345, Feb. 2012.

[68] A. Muller and J. Speidel, "Exact symbol error probability of M-PSK for multihop transmission with regenerative relays," IEEE Commun. Lett., vol. 11, no. 12, pp. 952-954, Dec. 2007. 